\title{
"FARE FILOSOFIA" E "FARE STORIA DELLA FILOSOFIA": DIVORZIO O CONVIVENZA?
}

\author{
Gregorio Piaia'
}

Università di Padova

\section{DESCRIZIONE}

Il presente articolo propone una riflessione sulle difficoltà relative alla distinzione tra "fare filosofia" e "fare storia della filosofia". Si sostiene la convenienza di questa distinzione problematica nella misura in cui la coesistenza delle due prospettive ne garantisce l'arricchimento reciproco. In particolare, si insiste sul fatto che la seconda prospettiva promuove un'attitudine che è più aperta alla comprensione dei modi distinti in cui l'essere umano ha cercato di accedere alla verità, e che evita l'autosufficienza in cui rischia di cadere la prima.

Keywords: Tipi di storia della filosofia; Comprensione; Ermeneutica.

\begin{abstract}
The present work proposes a reflexion about difficulties involved in the distinction between "doing philosophy" and "making history of philosophy". We support the convenience of this problematic distinction, but in a way that the coexistence of the two perspectives guarantees mutyal enrichment. In particular, we insist that the second perspective promotes an attitude that is more open to understanding the distinct ways in which the human being has tried to acces to the truth, and that avoids the self-sufficiency in which the first risks falling.
\end{abstract}

Keywords: Types of History of Philosophy; Understanding; Hermeneutics.

${ }^{1}$ gregorio.piaia@unipd.it 
Debbo confessare che l'invito a intervenire sul tema dei rapporti tra il "fare filosofia" e il "fare storia della filosofia" mi ha stimolato ma al tempo stesso imbarazzato. Non si tratta di un tema per me nuovo (il mio primo intervento in proposito risale al lontano $1974 \ldots)^{2}$ e d'altra parte le mie indagini nel campo della storia della storiografia filosofica mi offrono molti spunti di riflessione. Eppure, oggi ancor più di ieri, il tema continua ad apparirmi sfuggente per le sue varie sfaccettature e anche per il rischio sempre incombente di voler imporre una prospettiva che si fonda certo su una personale esperienza di ricerca, ma che finisce inevitabilmente col porsi in contrasto con altre esperienze personali, differenti ma pur sempre degne di rispetto e di attenzione. Questo imbarazzo dipende essenzialmente da tre motivi. Il primo e il più ovvio, direi quasi banale, è la grande quantità e varietà di discussioni che su questo tema si sono svolte da oltre tre secoli, per lo meno da quando il grande Leibniz prospettava l'esigenza di passare dalla tradizionale historia philosophorum di Diogene Laerzio ad una historia philosophiae imperniata sul confronto critico fra le dottrine e sulla loro logica "connessione". ${ }^{3} \mathrm{Ma}$, per limitarci alle dispute più lontane nel tempo, torna alla memoria anche lo scontro assai polemico (verso la metà del sec. XVIII) fra Johann Jacob Brucker e André-François Boureau-Deslandes sul modo d'intendere la storia "critica" della filosofia; ${ }^{4}$ oppure il Methodenstreit, la discussione sul "concetto" di storia della filosofia, cui nell'età kantiana presero parte Christian Garve, Karl Leonard Reinhold, Johann Christian August Grohmann, Johann Gottlieb Buhle ed altri ancora. ${ }^{5}$ Negli ultimi decenni la letteratura sull'argomento è diventata particolarmente ricca, anche se tutto sommato le posizioni di fondo non sono mutate di molto, al punto da constatare spesso che una certa posizione riecheggia, sia pure inconsapevolmente, posizioni che già erano state espresse parecchi anni prima e che ora vengono riscoperte e riproposte, come i corsi e ricorsi storici di Giambattista Vico.

A questo motivo d'imbarazzo se n'aggiunge un altro, rappresentato dalla natura intrinsecamente problematica dell'oggetto preso in esame dallo

\footnotetext{
2 Piaia 1975, 47-59.

3 Longo 1986, 43-52.

4 Piaia, Santinello (eds.) 2011.

${ }^{5}$ Geldsetzer 1966, 519-527.
} 
storico della filosofia: la filosofia, per l'appunto, o meglio le filosofie, data l'irriducibile mobilità del concetto di "filosofia". Il che rinvia a una domanda di fondo: in che misura il modo (o i modi) d'intendere oggi la filosofia condiziona il nostro modo di ricostruire i diversi modi in cui ieri o l'altro ieri è stato inteso e praticato il "far filosofia"? Detto in maniera più spiccia: che significa "far filosofia"? Ed ecco affiorare un'altra domanda: con quali criteri è stato di volta in volta definito il "canone" storico-filosofico e fino a che punto questo canone è valido ancora oggi? In altre parole: chi è che merita d'essere preso in esame dallo storico della filosofia e quali sono i filosofi cosiddetti maggiori, sui quali concentrare eventualmente la nostra indagine? Ma è poi storiograficamente legittima la distinzione tra filosofi maggiori e minori, frutto di una consuetudine che a prima vista può apparire neutrale ma che, gratta gratta, si rivela essere l'esito di ben precise scelte teoriche e di un'abile politica culturale? Basti pensare, ad es., ai "Magnifici Sette" che per lungo tempo hanno dominato nella ricostruzione della filosofia moderna da Descartes a Kant e che in realtà sono funzionali alla storiografia filosofica d'ispirazione kantiana: tre razionalisti (Descartes, Spinoza, Leibniz), tre empiristi (Locke, Berkeley. Hume) e poi ecco il filosofo di Königsberg, che con i giudizi sintetici a priori ha risolto la contrapposizione fra razionalisti ed empiristi... E dove mettiamo gli altri filosofi del Sei-Settecento, confinati a un ruolo di tappezzeria?

V'è pure un terzo motivo che sta al fondo del mio imbarazzo. Viviamo nell'era della globalizzazione culturale e non solo economica, che ha investito anche il modo di concepire la storia della filosofia (si veda, per tornare alla questione del canone, la denuncia dell'eurocentrismo che ha caratterizzato e caratterizza tuttora la storiografia filosofica). ${ }^{6}$ Eppure, quando si parla dei rapporti tra filosofia e storia della filosofia, non si può non tener presente l'incidenza delle diverse tradizioni nazionali e, all'interno di ognuna di esse, delle differenti "scuole". Queste tradizioni non sono certo monolitiche ed hanno anzi una loro dialettica interna, e tuttavia si presentano ad un occhio esterno con caratteri comuni e distinti da altre tradizioni nazionali. Si pensi, ad es., all'influsso esercitato in Italia da La filosofia come sapere storico (1959) di Eugenio Garin (che ha rovesciato la lunga egemonia neo-hegeliana di Benedetto Croce e Giovanni Gentile) e, per converso, al legame tra filosofia e storia della filosofia che caratterizza invece buona parte della produzione storiografica francese; oppure, guardando al panorama accademico italiano,

${ }^{6}$ Si veda, ad es., Park 2013; König-Pralong 2016. Si veda pure, con riferimento alla problematica attuale, Graness 2015, 78-96; Malik 2017; Elberfeld (ed.) 2017. 
si pensi alle cospicue riflessioni metodologiche condotte a Milano nell'ambito della scuola di Mario Dal Pra. ${ }^{7}$

Prima di entrare in medias res vorrei fare un'altra considerazione di carattere generale. Discutere sui rapporti tra filosofia e storia della filosofia significa fare riferimento a una più o meno esplicita "filosofia della storia della filosofia", cioè ad una prospettiva teorica che dal Seicento a metà Novecento è stata strettamente connessa al concetto di storia "generale" della filosofia, intesa non solo come un grande contenitore ma anche (e soprattutto) come una entità organica, unitaria e in continuo divenire, di cui dobbiamo cogliere il senso, la direzione o addirittura l'intrinseca legge di sviluppo. In sintesi: da dove viene, come procede e verso dove va la filosofia nel suo percorso storico unitariamente considerato ${ }^{8}$ Gli storiografi del XVII secolo, che iniziavano le loro trattazioni dalla "filosofia antidiluviana" o addirittura da Adamo (considerato primus parens et primus sapiens) e giungevano usque ad nostram aetatem, avevano le idee molto chiare al riguardo, e così pure gli storici d'ispirazione kantiana come Tennemann e Buhle, per non parlare di Hegel e dei suoi seguaci, che avevano un'idea ben precisa sulla "unità" della storia della filosofia. ${ }^{9}$ In realtà le domande di cui sopra comportano un aggancio con la più generale filosofia della storia quale si è sviluppata a partire dal Settecento, soppiantando la tradizionale visione teologica di matrice agostiniana che ispirava gli storici secenteschi della filosofia, come Georg Horn. ${ }^{10}$

Non è questa la sede per soffermarsi sulla crisi e sul ridimensionamento che la moderna filosofia della storia, fondata su un'idea forte di progresso, ha subito nel corso del Novecento. Interessa qui rilevare come alla pretesa (di volta in volta di matrice illuministica, hegeliana, positivistica, neohegeliana, marxista e così via) di "spiegare" nel suo complesso il cammino storico della filosofia si sia sostituita l'attenzione su singoli momenti o frammenti, rappresentati da un autore o da un tema oppure da uno dei tanti -ismi che a partire dal secolo XVIII costellano il discorso storico-filosofico. Ne consegue che le attuali grandi storie generali della filosofia sono in realtà un assemblaggio poco coordinato di contributi redatti da più studiosi. Ciò è

7 È emblematico a questo riguardo il saggio di Gianni Paganini (Paganini 2014, 137167), che prende avvio da riflessioni di carattere generale e nelle pagine conclusive si concentra su Mario Dal Pra e sui suoi allievi. Ma l'intero fascicolo di questa rivista offre spunti interessanti sui vari modelli nazionali di storiografia filosofica: si veda in particolare Pozzo 2014, 103-119 (sul "modello italiano"); Abbri 2014, 121-135; Cacciatore 2014, 275289. Sul modello francese: Ducat 2008, 139-158.

8 Garin 1990, 3-17.

9 Micheli, Santinello, Bianco, Longo 2015, 769-963; Santinello, 1995, 413-509.

10 Malusa 1993: 236-259. 
dovuto senza dubbio all'esigenza di ricorrere a competenze settoriali e specialistiche, ma rivela anche il venir meno di un disegno generale di quello che un tempo era unitariamente visto e ricostruito come il "cammino storico" della filosofia, con la conseguente de-costruzione di tale percorso. ${ }^{11}$

Un conto è però prendere atto di questa parabola che la storia generale della filosofia ha compiuto dagli inizi dell'età moderna fino ai nostri giorni, e farne oggetto di riflessione teorica e metodologica; un altro conto è liquidare frettolosamente la storia generale della filosofia riducendola a "dossografia", ossia ai manuali didattici (da Talete fino a Wittgenstein e Heidegger, per intenderci), come ha fatto Richard Rorty in un suo intervento sui "quattro generi della storiografia filosofica": uno cattivo (la "dossografia", per l'appunto) e tre buoni (la "ricostruzione razionale" propria della filosofia analitica, la "ricostruzione storica" tramite la contestualizzazione, la Geistesgechichte di Hegel, ripresa in vario modo da autori del Novecento da Heidegger a Reichenbach, Foucault, Blumenberg, MacIntyre). ${ }^{12}$ In realtà i manuali rappresentano soltanto la ricaduta didattica della storia generale della filosofia, sono cioè un suo sottoprodotto: un esempio classico è offerto dalle Institutiones historiae philosophiae usui academicae iuventutis adornatae (1748) che Johann Jacob Brucker ricavò dalla sua monumentale Historia critica philosophiae ab incunabulis mundi usque ad nostram aetatem deducta, apparsa a Lipsia negli anni 1742-1744 in 5 voll. in-quarto. ${ }^{13}$ Se, al di là degli schemi classificatori, utili ma spesso fuorvianti, prestiamo invece attenzione a quanto è storicamente avvenuto, non si può non riconoscere che anche le hegeliane Vorlesungen über die Geschichte der Philosophie si collocano nella linea di sviluppo delle grandi storie generali della filosofia del Sei-Settecento, che erano molto diverse dalle trattazioni (quelle sì erano dossografia!) di Teofrasto di Ereso, dei Vetusta placita e di Aezio. ${ }^{14}$ Il fatto che Hegel, pur utilizzandola come fonte, critichi e disprezzi la Historia critica philosophiae del Brucker non significa che quest'opera non avesse una sua prospettiva filosofica e fosse solo un ammasso di notizie ovvero di doxai: significa semplicemente che la filosofia cui si ispirava Brucker nel suo lavoro storiografico era diversa da quella cui si ispirava Hegel...15

11 Longo (ed.) 2003, 179-334 (in particolare i contributi di Mario Longo, Lutz Geldsetzer, Daniel Garber e Yves-Charles Zarka).

12 Rorty 1984, 49-75. Sulla prospettiva storiografica di Rorty: Vegas González 1993, $11-42 ; 30-37$.

13 Longo 2011, 547-549.

14 Cambiano (ed.) 1992, 307-416; Brancacci (ed.) 2005.

$15 \mathrm{Su}$ questo argomento mi permetto di rinviare al mio articolo, Piaia 2001, 69-81. 
Il dibattito su come operare in campo storico-filosofico è dunque sempre aperto e si potrebbe anzi dire che sia costitutivo di una disciplina bifronte qual è la storia della filosofia, ove il genitivo "della filosofia" può essere inteso in un duplice modo: in senso strettamente oggettivo (la filosofia del passato è oggetto di ricostruzione storica, al pari di qualsiasi altra attività umana, teorica o pratica che sia) oppure in senso anche soggettivo, e in questo caso è la filosofia stessa che fornisce i criteri e i metri di giudizio per interpretare il suo passato. La filosofia, dunque, che fa la storia di se stessa: ma si tratta della Filosofia in sé e per sé o, assai più modestamente, della mia filosofia ovvero del mio modo d'intendere il "far filosofia"? Di qui, volendo schematizzare, due diversi modi di accostarsi al passato della filosofia: un modo storico e un modo speculativo.

Nella prima modalità di approccio l'obiettivo è di studiare Aristotele o Kant o qualsiasi altro filosofo "nel loro tempo"; il che, sia chiaro, non significa solo definire le date esatte di nascita e di morte di questi personaggi, tracciare la loro biografia, elencare le opere e riassumerne le dottrine come nella historia philosophorum del buon Diogene Laerzio. Si tratta anzitutto di indagare sulle tradizioni speculative con cui questi pensatori furono in contatto (è il problema delle fonti), in modo da chiarire la loro effettiva originalità rispetto ai contesti culturali in cui vissero e operarono. Si tratta poi d'indagare sull'evoluzione o meno del loro pensiero, sulla coerenza interna delle diverse fasi di tale pensiero e sulla sua fortuna, cercando anzitutto, per quanto sia possibile, di leggere e interpretare Aristotele con Aristotele e Kant con Kant, ossia di analizzare la genesi e il vario articolarsi delle loro teorie, nonché i problemi ch'essi lasciarono aperti o insoluti. Va da sé che una qualche attrazione o sensibilità personale per le teorie di Aristotele o di Kant si dovrà pur avere da parte dello storico della filosofia, a meno che non sia affetto da una forte dose di autolesionismo o masochismo (!). Infatti egli può sentirsi in sintonia con certe enunciazioni di questi autori e con il loro modo di accostarsi ai problemi filosofici e di viverli, come pure dissentire da certe loro conclusioni; ma ciò non pregiudica l'obiettivo sopra indicato della comprensione attraverso la contestualizzazione, per cui le convinzioni personali dello storico dovrebbero restano sullo sfondo o sotto traccia.

Nell'altra modalità di approccio posso rivolgermi ad Aristotele o a Kant o a qualsiasi altro pensatore muovendo da un problema filosofico che mi sta a cuore (ad es. il concetto di "categoria" oppure la nozione di "legge morale") per verificare se l'impostazione teorica e l'attrezzatura concettuale adottate da questi autori mi possano essere d'ausilio per giungere alla chiarificazione o a una qualche soluzione di tale problema. Per questa indagine mi servo di un criterio o parametro o modello che ho elaborato sul piano teorico e che 
posso quindi applicare ad Aristotele come a Immanuel Kant o a George Edward Moore. Da questo punto di vista la mia attenzione va non tanto a ciò che Aristotele o Kant intendono per "verità" e alle ragioni - nonché alle circostanze - che li hanno condotti su una certa via, quanto al tasso di "verità in sé" (che è poi, a dire il vero, la "verità per me"...) racchiuso in una determinata formulazione dottrinale, ovvero la maggior o minore capacità di reagire positivamente al modello adottato. Che poi tale formulazione sia uscita dallo stilo di Aristotele o dalla penna d'oca di Kant o dal personal computer di un professore universitario della Nuova Zelanda (e quindi da contesti culturali assai diversi fra loro) è alla fin fine irrilevante dal punto di vista speculativo, ossia agli effetti del mio guadagno teorico.

Sono significative, a questo riguardo, le considerazioni di Willard Van Orman Quine sulla sua frustrante esperienza quale docente di storia della filosofia all'Università di Harvard, quando, anziché spiegare quale fosse effettivamente il pensiero di David Hume, egli avrebbe preferito illustrare ai suoi studenti se e quanto fosse in sé vera una proposizione tratta dagli scritti del pensatore scozzese. ${ }^{16}$ Certo, non c'è da meravigliarsi se Quine, che è considerato la quintessenza del classico filosofo analitico, mostra ben poco interesse per l'operazione storico-filosofica. Se però ci spostiamo sul versante opposto, quello metafisico, troviamo non a caso un atteggiamento analogo. Il Seicento è il secolo in cui si afferma la historia philosophica, eppure un filosofo indubbiamente speculativo, come Malebranche, oppone nettamente il fatto storico alla verità filosofica, dicendo chiaro e tondo che coloro che si affaticano sui libri per interpretare le dottrine di Platone o di Aristotele "non sanno che storie e fatti, e non verità evidenti; e sono piuttosto degli storici che dei veri filosofi, uomini che non pensano affatto ma che sono in grado di raccontare i pensieri altrui". E ancora, per la gioia di quanti sono soliti denunciare i limiti o la scarsa utilità dell'approccio storico alla filosofia:

Mi sembra che sia abbastanza inutile, per quelli che vivono presentemente, sapere se vi sia mai stato un uomo che si chiamasse Aristotele, se quest'uomo abbia scritto [effettivamente] i libri che portano il suo nome, se egli intenda una tal cosa o un'altra in un tal luogo delle sue opere; ciò non può rendere un uomo né più saggio né più felice, ma è molto importante sapere se quanto dice sia vero o falso in sé. È pertanto inutile sapere ciò che Aristotele ha creduto sull'immortalità dell'anima, benché sia molto utile sapere che l'anima è immortale. ${ }^{17}$

Messi insieme, i giudizi di Quine e di Malebranche sembrano rappresentare una pietra tombale per l'attività storico-filosofica e, se coerentemente applicati, porterebbero alla totale espulsione dei professori di storia

\footnotetext{
16 Quine 1985, 194.

17 Malebranche 1946, 151 e 154.
} 
della filosofia dai Dipartimenti di Filosofia. Ma qui si avverte subito il bisogno di una chiarificazione, che riguarda l'atteggiamento filosofico di chi fa storia della filosofia. Nel caso dell'approccio storico non si chiede che l'operatore rinunci ad avere una sua filosofia o sia più uno storico che un filosofo (è questa l'accusa che viene generalmente mossa), ma gli si chiede piuttosto che abbia verso le filosofie del passato un atteggiamento di curiosità e di apertura non solo a ciò che è simile al nostro modo di pensare, ma anche e soprattutto al "diverso", all"altro da noi": un atteggiamento non certo nuovo, icasticamente espresso nella celebre sentenza di Terenzio (Homo sum, humani nihil a me alienum puto), ma ben presente, ad es., nelle riflessioni di uno spirito libero e moderno come Montaigne. ${ }^{18}$

Questo tema merita a mio avviso una sottolineatura, poiché il lavoro storico-filosofico, al pari di ogni lavoro storico, richiede un affinamento della nostra capacità di 'comprendere' (il Verstehen di Dilthey), nel senso in cui è stata analizzata da Isaiah Berlin in una lecture risalente al 1953 ma pubblicata solo sul finire del secolo. ${ }^{19} \mathrm{Si}$ tratta cioè di puntare alla comprensione dell'alterità del testo filosofico, che rinvia a sua volta all'alterità personale dell'autore e che lo storico della filosofia, proprio in quanto operatore storico, cerca di cogliere con un delicato e complesso lavoro di analisi filologica e di contestualizzazione, quasi uscendo da sé per farsi vicino all'altro. Questo nesso filosofia-persona non rinvia unicamente a un complesso di dati biografici e ambientali - il cosiddetto inquadramento storico - che fungono da semplice contorno alle posizioni speculative, sulle quali andrebbe invece concentrato lo sforzo di verifica logico-concettuale. Anche la persona del filosofo, e di conseguenza le sue scelte intellettuali, partecipano di quei "due livelli" che coesistono in ognuno di noi:

[...] una superficie superiore, pubblica, illuminata, facilmente rilevabile e nitidamente descrivibile, da cui è possibile estrarre proficuamente delle somiglianze $\mathrm{e}$ condensarle in leggi; e al di sotto di essa un sentiero che conduce a caratteristiche via via meno ovvie e ciò nondimeno più intime e pervasive, troppo strettamente mescolate con i sentimenti e le attività perché sia agevole distinguerle da questi. ${ }^{20}$

Viene allora da chiedersi se le teorizzazioni di un filosofo siano risolvibili nella consequenziale e asettica linearità di un procedimento logico o se

18 Montaigne 1982, 301: "Io non incorro affatto nel comune errore di giudicare un altro secondo quello che sono io. Ammetto facilmente cose diverse da me. Per il fatto di sentirmi impegnato in una certa forma, non vi obbligo gli altri, come fanno tutti; e immagino e concepisco mille contrarie maniere di vita, e diversamente dalla gente comune, noto più facilmente la differenza che la somiglianza (et, au rebours du commun, reçoy plus facilement la difference que la ressemblance en nous)".

19 Berlin 1998, 29-84.

20 Berlin 1998, 57. 
non dipendano in qualche modo anche da "questo livello di abitudini articolate solo a metà, di presupposti e modi di pensare non analizzati, di reazioni semi-istintive, di modelli di vita così profondamente radicati da non affiorare affatto alla coscienza". ${ }^{21}$ Non si tratta di riproporre uno psicologismo a buon mercato, con l'ennesimo e scontato richiamo al subconscio. Quella che è in questione, daccapo, è la nostra capacità di comprendere l'altro', ossia di sviluppare quel sense of reality che per Berlin non si raggiunge con le scienze sociali, bensì con il pascaliano esprit de finesse, di cui sono maestri - sempre secondo Berlin - i grandi storici e i grandi politici. È pertanto vana la pretesa di trovare "qualche infallibile chiave scientifica" per risolvere il problema della comprensione dell'uomo e delle sue produzioni intellettuali, prima fra tutte la filosofia. "Possiamo solo, nel migliore dei casi - nota Berlin con una punta di ironia - indulgere all'arte di una squisita pittura di ritratto (the art of exquisite portrait-painting)". ${ }^{22}$ Domanda provocatoria: che sia questa l'alternativa post-moderna alla concezione teoreticamente forte della storia della filosofia, proposta da Hegel e variamente ripresa dai suoi eredi?

Volendo ora etichettare con un -ismo l'atteggiamento di apertura all'altro che contraddistingue chi fa storia della filosofia, si potrebbe parlare di un mix di eclettismo e di moderato scetticismo: una sorta di eclettismo scetticheggiante, ove l'onesta consapevolezza dell'impossibilità di raggiungere in toto la verità e di poterla quindi esibire non dà luogo a un atteggiamento negativo di rifiuto o di indifferenza, ma a un habitus di apertura alle svariate e molteplici espressioni dell'umano pensare; per cui, ad es., l'interesse per s. Agostino o per Pascal o per Kierkegaard non esclude affatto un pari interesse per Voltaire e Lamettrie o persino per l'autore de La philosophie dans le boudoir, il "divino" marchese de Sade. Mi rendo conto che nel comune sentire filosofico il concetto di eclettismo non gode di buona fama, in quanto sinonimo di scarso rigore e di scarsa consistenza teorica; e qui la memoria va subito al vituperato éclectisme di Victor Cousin e alla sua pretesa di costruire con il metodo eclettico un "sistema" di filosofia più inclusivo e al tempo stesso più rigoroso degli altri sistemi. ${ }^{23}$ Non è però all'eclettismo ottocentesco di Cousin che faccio qui riferimento, bensì alla settecentesca philosophia eclectica di Christian Thomasius, di Franz Budde, di Johann Jacob Brucker, di Denis Diderot, di Antonio Genovesi e di molti altri autori, che in nome della libertas philosophandi si opponevano alla philosophia sectaria di chi, chiudendosi all'interno di una scuola ben definita, criticava e respingeva $a$ priori le altre scuole, com'era il caso della tradizionale Scolastica (contro la

\footnotetext{
21 Berlin 1998, 57.

22 Berlin, 58.

23 Piaia 2004, 152-162.
} 
quale a suo tempo Erasmo da Rotterdam aveva lanciato i suoi strali satirici) ${ }^{24}$ ma anche del recente cartesianismo. ${ }^{25}$ Era questa, a ben vedere, la lezione offerta a cavallo del 1700 da un singolare historien-philosophe quale Pierre Bayle, cui non a caso si richiamò Eugenio Garin in una pagina che a quasi mezzo secolo di distanza appare quanto mai viva e merita ancor oggi d'essere oggetto di riflessione:

In realtà è proprio quest'abito di tolleranza intellettuale, unito all'idea di una pluralità di punti di vista possibili, che alimenta una storia che vuole conoscere con estrema fedeltà le varie posizioni. Se la verità è, in quanto raggiungimento definitivo, le désespoir così dello storico come del filosofo; se la conoscenza è una ricerca più o meno plausibile, ma non mai conclusa, si giustifica non solo l'indugio curioso su tutte le ricerche, ma l'ansia puntigliosa di indagarle tutte sino in fondo, di coglierne fin dove è possibile il senso, di scandagliarle nei particolari e di saggiarne la consistenza criticandole. ${ }^{26}$

Il désespoir de la vérité, che di primo acchito suona come una resa scettica di fronte a una battaglia impossibile a vincere, va però inteso nella sua valenza positiva. Al pari della letteratura e delle arti, anche la filosofia contribuisce ad arricchire il patrimonio umano (i cosiddetti beni culturali) e i suoi prodotti non finiscono nel "cimitero delle opinioni", ma costituiscono senza dover necessariamente ricorrere al tocco magico della dialettica hegeliana - altrettante testimonianze dello sforzo umano verso la verità: una sorta di grande caleidoscopio filosofico, in cui i singoli frammenti più meno estesi, colti e rivissuti nel loro contesto, non riescono a comporsi in una unità organica per la semplice ragione che tendono alla verità, in sé inesauribile, da angolature fra loro diverse, cioè da differenti contesti storici e dottrinali e da differenti prospettive personali. Non resta che prendere atto di tale umana condizione e godere di questi frammenti (benché ci appaiano parziali o strani o addirittura inutili) attraverso il contatto con i testi che ce ne hanno trasmesso la memoria.

Diverso è il caso di chi, nel suo relazionarsi alle filosofie del passato, muove da una ben definita prospettiva teorica, cosa assai allettante e gratificante, ma che apre la via a questioni di non poco conto, che non riguardano solo gli esiti finali di questo tipo d'indagine. Supponiamo, ad es., che l'operatore muova da una prospettiva oggi assai diffusa qual è quella logico-epistemologica. I criteri di giudizio che ne conseguono funzionano egregiamente

24 Erasmo da Rotterdam 1997, 173: "Queste sottilissime sottigliezze [subtilitates] sono ulteriormente assottigliate dai numerosi sistemi della scolastica, per cui ti districherai prima dal Labirinto che dai grovigli dei Realisti, dei Nominalisti, dei Tomisti, degli Albertisti, degli Occamisti, degli Scotisti, e non sono nemmeno tutte le sètte ma solo le principali".

25 Longo 1998, 52-65. Sulla varia vicenda dell'eclettismo filosofico: Albrecht 1994.

26 Garin 1993], 266. 
nel caso dei pensatori sopra menzionati (Aristotele, Kant, Moore), ma ho qualche dubbio sulla loro applicazione a pensatori come s. Agostino, Kierkegaard o Nietzsche. Questo significa forse che Aristotele, Kant, Moore sono "veri" filosofi a differenza di un Agostino o di un Kierkegaard o di un Nietzsche, i cui contenuti teorici, in quanto poco "rigorosi", sarebbero meno interessanti e nei cui confronti ci si mostra alla fin fine indifferenti? In questo caso l'interesse esclusivo per certi contenuti teorici, giudicati tali in base a un criterio di tipo epistemologico, porterebbe alla rimozione o quanto meno all'emarginazione di parecchi autori dal canone dei filosofi e quindi dal panorama degli studi storico-filosofici. Ma questa non sarebbe, tutto sommato, una forma di razzismo intellettuale?

Queste differenti impostazioni del rapporto con il passato filosofico hanno dato luogo, e continueranno a dar luogo, a reciproche accuse e recriminazioni, per cui gli uni sono spesso inclini a denunciare come una manchevolezza l'assenza di un adeguato spessore filosofico, gli altri a negare con tono deciso che certe trattazioni abbiano carattere storico-filosofico, distinguendo nettamente il "far filosofia" dal "far storia della filosofia". Prima ancora che in consapevoli e meditate scelte teoriche e metodologiche, questo persistente conflitto fra storici e teoreti ha le sue radici in differenti vocazioni e sensibilità intellettuali, per cui parrebbe sensato ed opportuno, dopo tante baruffe in famiglia, sancire una definitiva separazione, anzi un vero e proprio divorzio, fra l'approccio speculativo - la storia "filosofica" della filosofia - visto come un momento preparatorio del lavoro teoretico (oggi è infatti difficile, anche per gli stessi filosofi analitici, "far filosofia" senza un confronto critico con il pensiero del passato), ${ }^{27}$ e l'approccio più propriamente storico, ormai assimilabile o accostabile alla tradizionale storia delle idee o alla più recente Intellectual history o alla Begriffsgeschichte oppure a quel settore della Kulturgeschichte che è la storia della cultura filosofica. ${ }^{28}$ Dopo tre secoli di grande sviluppo e di forti oscillazioni teoriche e metodologiche (riecco la "filosofia della storia della filosofia"!), l'attività storico-filosofica perderebbe così la sua esaltata quanto contrastata autonomia disciplinare scindendosi in due tronconi: da una parte il rientro nel grande alveo materno della filosofia, dall'altra l'emigrazione in un territorio dominato dal rapporto fra storia e scienze sociali. Finis historiae philosophiae?

27 Vienne (ed.) 2005); Jaran 2011, 171-192; Reck (ed.) 2013.

28 Lovejoy 1940, 3-23; Mandelbaum 1965, 33-66; Viano 1966, 255-261; Piovani 1965; King (ed.) 1985; Richter 1987, 247-263; Collini 1985; Bianchi (ed.), 1989; Tessitore 1990; Tessitore 2014, 3-29; Scholtz 1996, 133-151; Cacciatore 2001, 205-224; Grafton 2006, 1-32; Anheim, Lilti et Van Damme 2009, 5-11; Biris 2012, 28-35; Hutton 2014, 925937. 
Per quanto seducente, una simile soluzione presenta tuttavia notevoli controindicazioni, perché, venuto meno il continuo confronto (sia pure armato) e il reciproco stimolo tra i diversi fruitori del passato filosofico, essa finirebbe con l'accentuare i difetti dell'uno e dell'altro metodo di approccio, come già rilevava con grande lucidità Antonio Rosmini, il maggiore filosofo italiano del primo Ottocento, in uno scritto composto intorno al 1826, in piena età hegeliana. ${ }^{29} \mathrm{Ma}$ è anche facile obiettare che, insistendo nel mantenere i rapporti tra il far filosofia e il fare storia della filosofia, si corre il rischio di produrre sia della cattiva filosofia sia della cattiva storiografia, come sottolineava Eugenio Garin in una breve nota che risale a molti anni fa ma che suona ancora attuale. ${ }^{30}$

Questo rischio è sempre incombente; tuttavia, al di là delle divisioni astratte e delle ripartizioni disciplinari ufficiali, occorre anche tener conto di quella zona di frontiera (una sorta di terra di tutti e di nessuno) in cui è difficile distinguere nettamente l'approccio storico da quello teorico, che risultano spesso fusi insieme o comunque interconnessi. La qual cosa può certamente dare luogo a confusioni ed equivoci (ecco la "cattiva storia" e la "cattiva filosofia"), ma è anche vero che l'interazione fra questi due àmbiti di ricerca si è spesso rivelata feconda malgrado le inevitabili contaminazioni o forse proprio grazie ad esse, per una sorta di eterogenesi dei fini applicata al mondo delle idee. Si pensi, ad es., alla celebre opera di Ernest Renan, Averroès et l'averroïsme (Paris 1852), che segnò una svolta fondamentale negli studi sulla filosofia islamica e sui suoi rapporti con l'Occidente latino: essa era certamente frutto delle competenze linguistiche, filologiche e storiche di un grande semitista, il quale però non avrebbe scritto quest'opera (o l'avrebbe scritta in maniera molto diversa) se non fosse stato pervaso da una

29 Rosmini-Serbati 1883, 53-54: "Senza la filosofia la storia è cieca e fassi un noiosissimo andirivieni dello spirito umano, una successione di opinioni tutte di egual peso, o piuttosto di egual leggerezza, senza che si distingua giammai l'una sentenza alle altre preferirsi con ragione. Senza la storia, la filosofia diventa così secca, così gratuita, così lontana dalle forze dell'ingegno, che non può ch'essere ricevuta sterilmente dalla memoria, e giacere in essa come un penoso ingombro, ovvero un semenzaio di dubbi e di inquietudini interminabili [...]. La storia dunque si può dire il veicolo della filosofia; la filosofia all'incontro può dirsi la luce della storia".

30 Garin 1971, 340-342, ove si rileva come la ricerca storico-filosofica, "dopo un periodo di furori polemici, è tornata a volte a ricollocarsi nell'alternativa fra erudizione deserta di idee e commisurazione di un passato convenzionalmente ricostruito in base a non originali letture, a schemi teorici generali assunti a priori. Di qui alquante discussioni metodologiche a vuoto, un po' di intellectual paleontology, qualche dose di erudizione sempre più minuta, e alla fine il gioco del vivo e del morto, dei rami secchi e delle fronde verdi tutte eguali, del filosofo grande e piccolo, traviato o no, dell'analisi dottrinale o philosophia superior opposta alla ricerca detta filologica o philosophia inferior. Ossia, a ben vedere, cattiva storia e cattiva filosofia" (342). 
forte prospettiva filosofica, ispirata a una visione decisamente laica e progressista della storia e della cultura. Spostandoci sull'opposto versante ideologico, si pensi agli sviluppi eccezionali assunti dalla storiografia sul pensiero medievale con l'avvento della Neoscolastica; ma senza l'enciclica Aeterni Patris (1879), che non era certo ispirata dalle istanze metodologiche della "scuola storica", assai vicine al positivismo, difficilmente sarebbe sorto uno studioso del calibro di Martin Grabmann... Oppure si pensi ai numerosi e considerevoli contributi prodotti - in particolare in Italia - dalla storiografia filosofica d'ispirazione marxista, nonostante il suo palese taglio ideologico, che talora si è tradotto in uno smaccato uso politico della storia della filosofia. ${ }^{31}$ Ma riguardo a questa stagione "militante" della storiografia filosofica vale la pena citare nuovamente Eugenio Garin, che, pur aderendo sul piano politico al Partito Comunista Italino, a metà degli anni Settanta (e quindi alla vigilia dei cosiddetti "anni di piombo", segnati dal terrorismo dell'ultrasinistra) mise in guardia contro un uso improprio e metodologicamente scorretto di alcune tesi di fondo del marxismo, applicate alla storia delle idee:

Se è vero [...] che "la produzione intellettuale si trasforma insieme con quella materiale", una corretta analisi ravvicinata della prima gioverà assai più di accostamenti estrinseci fra aspetti mal compresi dell'una e momenti dell'altra afferrabili solo con strumenti adeguati e competenze specifiche, e non attraverso semplici analogie di superficie. Non a caso, a volte, pretese spiegazioni "profonde", strutturali, di mutamenti di idee morali, filosofiche, religiose, politiche, fanno pensare solo alla chiamata in causa di un "dio ascoso", inconoscibile, e perciò sconosciuto. ${ }^{32}$

D'altro canto lo stesso fossato che separa l'approccio storico ("Comprendere Kant nel suo tempo") dall'approccio teoretico ("Che cosa può dirci Kant in ordine a un certo problema attuale?") appare meno profondo se si tiene presente che fra questi due ambiti si è venuto costituendo un ambito in qualche maniera intermedio. Si tratta del complesso di interpretazioni di cui un pensatore è stato oggetto nel corso del tempo (la storia della sua fortuna e della sua critica), cui di solito si dà solo un peso marginale, nella presunzione di poter stabilire, tramite i testi in lingua originale, un rapporto "diretto" con l'autore. In realtà questo presunto rapporto diretto è variamente arricchito - ma anche influenzato e talora condizionato - dagli strati interpretativi che nel corso del tempo (si pensi a Platone e al "platonismo") si sono depositati sul pensiero originario di un autore, stabilendo una sorta di continuità tra lo studioso e l'oggetto della sua ricerca: una continuità ricca

31 Si veda in particolare Badaloni 1964, 62-86; Dal Pra 1996, 95-110; Luporini 1974; Merker 1974, 13-47.

32 Garin 1996. 
di aspetti storico-culturali ma anche di suggestioni e implicazioni teoriche, della cui influenza non sempre lo studioso si mostra avvertito.

Ma v'è soprattutto un motivo che ci induce a non abbattere il pur esile ponte che tiene ancora in collegamento il "fare storia della filosofia" con il "fare filosofia". Nella misura in cui l'indagine storiografica, muovendo dalle sue basi filologiche e utilizzando il metodo della contestualizzazione storicoculturale, ci porta a stabilire un contatto con un autore del passato e quindi a "comprendere", per quanto ci è possibile, il suo pensiero e, in ultima analisi, a dialogare e a con-filosofare con lui, lo storico della filosofia compie un'operazione che riguarda anche il far filosofia. Anzi, questa operazione si configura come uno dei possibili modi di far filosofia: una philosophia minor, se si vuole, giacché questo far rivivere in noi un pensiero altrui, cercando però di rispettare le intenzioni dell'autore e il suo contesto, esclude certamente la produzione di teorie nuove, ma è pur sempre un'operazione intellettuale e non meramente dossografica, che non è affatto priva di ricadute esterne; e questo perché il lavoro interpretativo dello storico dovrebbe indurre anche il teoreta (nell'ipotesi che egli legga i lavori dello storico...) a rivedere o a meglio calibrare i propri schemi di lettura e quindi i propri nessi concettuali.

Il rapporto tra filosofia e storia della filosofia non si risolve dunque nella schematica contrapposizione fra la pura e semplice indagine filologica e storica, da una parte, e, dall'altra, la forzata assimilazione di un pensatore al punto di vista dell'interprete. Esso assume invece la forma del circolo ermeneutico, che si realizza nel rapporto sempre più profondo fra il soggetto che interroga e il testo che è interpellato. Ed ecco ritornare in primo piano, contro gli eccessi di un facile ermeneutismo oggi di moda, il tema dell'alterità del testo e del suo autore, che nella maggioranza dei casi non è più in vita ma continua a vivere e a parlarci attraverso le sue opere. Riguardo a questo rapporto interpersonale restano quanto mai attuali le considerazioni che Luigi Stefanini, il filosofo padovano del personalismo, fece a un convegno svoltosi nel lontano 1952:

Il colloquio con il passato è un caso particolare del colloquio senz'altro, e ne segue le leggi. Non intendo chi attualmente mi parla, se non riesprimo da me le sue parole. Le intendo in quanto le faccio mie, ma non intenderei chi mi parla e vaneggerei solitario, se ciò che riesprimo da me non fosse riespresso col segno dell'appartenenza all'altro, che mi precede e condiziona nell'espressione. La parola dell'altro mi precede di un attimo, ma, se mi precedesse di un millennio, il rapporto sarebbe identico. ${ }^{33}$

${ }^{33}$ Stefanini, 1962, 63. 
Inevitabile, a questo punto, il richiamo a Friedrich Schleiermacher e alla sua rifondazione dell'ermeneutica in quanto arte del comprendere il discorso altrui in modo progressivo, anche se mai definitivo. Rileggiamo, ad es., l'inizio delle sue Lezioni del 1832-1833, tutto incentrato sul rapporto interpersonale e non sull'astratto confronto di concetti e categorie:

Il compito dell'interpretazione psicologica considerato in sé e per sé è, in generale, quello d'intendere ogni complesso dato di pensiero come momento vitale di un uomo determinato (als Lebensmoment eines bestimmten Menschen). Che mezzi abbiamo per risolvere questo compito? Dobbiamo risalire al rapporto tra colui che parla e colui che ascolta. Quando il pensare e la connessione dei pensieri sono in entrambi gli interlocutori una sola e medesima cosa, allora, a parità di lingua, la comprensione (das Verstehen) va da sé. Quando invece il pensare è essenzialmente diverso in entrambi, la comprensione non va da sé, anche a parità di lingua. Se assolutizziamo i due casi, il compito scompare. Infatti, il compito non sorge affatto nel primo caso, dal momento che coincide completamente con la risoluzione; nel secondo è, a quanto pare, irrisolvibile. Ma in questa rigidezza o assolutezza è del tutto assente l'opposizione. Infatti in ogni caso è sempre presente una certa differenza di pensiero tra colui che parla e colui che ascolta, la quale non è però irrisolvibile. Anche nella vita di tutti i giorni, quando, a perfetta parità e trasparenza di lingua, ascolto il discorso di un altro e mi pongo il compito di comprenderlo, stabilisco una differenza tra lui e me. Ma in ogni volontà di comprendere un altro si presuppone già che la differenza sia risolvibile. Il compito consiste nell'entrare con più precisione nella natura e nei fondamenti (in die Beschaffenheit und Gründe) delle differenze tra colui che parla e colui che comprende. Ciò è difficile. ${ }^{34}$

Non è qui il caso di soffermarci sulle distinzioni svolte da Schleiermacher fra lingua e pensiero, e quindi fra interpretazione "grammaticale" od oggettiva e interpretazione "tecnica" o soggettiva. Va semmai ricordato come lo sviluppo dell'ermeneutica in senso accentuatamente filosofico ad opera di Heidegger abbia rotto il delicato ma imprescindibile equilibrio fra queste due modalità di approccio e fra il metodo "divinatorio" e quello "comparativo", trasformando la lettura del passato in un'operazione in cui l'istanza teorizzante prende decisamente il sopravvento, con esiti esaltanti quanto discutibili (l'“ermeneutismo" sopra menzionato, per cui il lettore 'trova' nel testo ciò che vi mette dentro di suo...). Vorrei soltanto ribadire che i due metodi rinviano l'uno all'altro e non sono quindi separabili, e che l'immedesimazione" con l'autore è solo una meta ideale (una sorta di kantiana idea regolativa), poiché il processo di avvicinamento all'altro non è mai esaustivo e non giunge mai alla reciproca "fusione delle anime", con tutto l'alone sentimentale che circonda tale espressione. ${ }^{35}$

34 Schleiermacher 1996, 490-491.

35 Longo 1995, 212-220 e 250-262. Si veda in particolare 214: "Pur conservando il principio (rivendicato da F. Schlegel e da Novalis) del collegamento con la filosofia, Schleiermacher difende il carattere autonomo della filologia, e lungi dal farne un semplice surrogato della filosofia, la intende come lo strumento per conferire a questa concretezza e 
Le considerazioni e i distinguo sin qui svolti non annullano dunque il divario tra i due modi (storicizzante e teoretizzante) di accostarsi al pensiero del passato, giacché tale divario, come s'è detto, nasce anzitutto dalla sensibilità e dalla storia personale dello studioso, cioè dal profondo del nostro io, prima ancora che da un'opzione metodologica. Dietro le più raffinate tecniche di approccio a un testo filosofico vi è pur sempre una "persona", nella complessa integralità delle sue componenti (e qui mi richiamo alla distinzione di Berlin fra i "due livelli"). Va quindi riconosciuta la specificità del lavoro di storicizzazione/contestualizzazione, e quindi la sua piena autonomia, contro la prospettiva ancillare di chi ancor oggi vede in esso solo un momento propedeutico al lavoro più squisitamente speculativo. Questo riconoscimento dovrebbe però accompagnarsi alla serena consapevolezza che la distinzione di metodi e di ruoli non comporta un radicale distacco tra il "fare filosofia" e il "fare storia della filosofia", poiché distinguere non equivale a separare; anzi, proprio il "distinguere" serve ad evitare - questa volta il richiamo a Hegel non è critico - che il nostro rapporto con le filosofie e i filosofi del passato si riduca a una "notte senza luna, in cui tutte le mucche appaiono bigie".

Resta pertanto valida (e qui rifà capolino la tradizione filosofica nazionale...) l'istanza di Giambattista Vico, che vedeva filologia e filosofia insieme connesse, nello sforzo di leggere, rivivere, gustare e mettere a frutto quelli che nel sec. XVIII erano chiamati "i prodotti dell'umano ingegno". Non più un divorzio, dunque, bensì una convivenza, non certo facile ed anzi problematica e spesso conflittuale, con i suoi momenti sereni (in cui ognuno svolge il suo lavoro) e i suoi momenti di crisi; ma la crisi, se pensiamo alla sua etimologia, non è in sé e per sé negativa: è un momento di confronto, di scelta e quindi di mobilitazione intellettuale. Ed anche questo, a ben vedere, rientra in qualche modo nel "far filosofia", se vogliamo evitare la tentazione del solipsismo e dell'autosufficienza.

realtà. La filologia è dunque filosofica anche per Schleiermacher, ma non nel senso della sistemazione e deduzione del suo contenuto da verità superiori e astratte, bensì, come è stato detto con efficacia, nel senso 'dell'auscultazione della gloria dell'essere nell'individuo' (Moretto 1979, 336), sulla base non dell'uso delle categorie logiche ma della categoria ermeneutica della comprensione". 


\section{BIBLIOGRAFIA}

Abbri, Ferdinando. 2014. "Tra indifferentismo e contestualismo: filosofia, scienza e cultura nel panorama storiografico nordamericano". Philosophia 10-11: 121-135.

Anheim, Étienne; Lilti, Antoine; Van Damme, Stéphane. 2009. “Quelle histoire de la philosophie?". Annales. Histoire, Sciences sociales: 5-11.

Albrecht, Michael. 1994. Eklektik. Eine Begriffsgeschichte mit Hinweisen auf die Philosophie-und Wissenschaftsgeschichte. Stuttgart - Bad Cannstatt: Frommann-Holzboog.

Badaloni, Nicola. 1964. "Filosofia, storia, e storia della filosofia nel marxismo". Rivista critica di storia della filosofia 19: 62-86.

Berlin, Isaiah. 1998. Il senso della realtà. Studi sulle idee e la loro storia. Milano: Adelphi.

Bianchi, Massino L. (ed.). 1989. Storia delle idee: problemi e prospettive. Firenze: Olschki.

Biris, Ioan. 2012. "The History of Thinkers or the History of Ideas? Michael Dummett's Model". Procedia - Social and Behavioral Sciences 71: 28-35.

Boucher, David. 1985. Texts in Context. Revisionist Methods for Studying the History of Ideas. Dordrecht: Reidel.

Brancacci, Aldo (ed.). 2005. Philosophy and Doxography in the Imperial Age. Firenze: Olschki.

Cambiano, Giuseppe (ed.). 1986. Storiografia e dossografia nella filosofia antica. Torino: Tirrenia.

—. 1992. "La doxographie antique". Revue de Métaphysique et de Morale 97: 307-416.

Cacciatore, Giuseppe. 2001. "La storiografia filosofica italiana tra storia delle idee e storia della cultura". Rivista di storia della filosofia 56: 205-224.

—. 2014. "Filosofare dopo Ortega: su alcuni modelli di storia della filosofia e storia delle idee nella Spagna della seconda metà del Novecento". Philosophia 10-11: 275-289.

Collini, Stefen. 1985. "What is Intellectual History?". History Today 35-10: https://www.historytoday.com/stefan-collini/what-intellectual-history

Dal Pra, Mario. 1996. "Materialismo storico e storiografia filosofica". In Mario dal Pra, Storia della filosofia e della storiografia filosofica. Milano: F. Angeli: 95-110.

Ducat, Philippe. 2008. "L'historicité de la philosophie et son enseignement : l'exemple français". Revue philosophique de Louvain 106-1: 139-158.

Elberfeld, Rolf (Hg.). 2017. Philosophiegeschichtsschreibung in globaler Perspektive (Deutsches Jahrbuch Philosophie 9-2).

Garin, Eugenio. 1971. "Discussioni di storiografia filosofica”. Rivista critica di storia della filosofia 26: 340-342.

—.1974. Intellettuali italiani del XX secolo. Roma: Editori Riuniti. 
—. 1990. "L"unità' nella storiografia filosofica". In Garin, Eugenio (ed.), La filosofia come sapere storico. Con un saggio autobiografico. Roma-Bari: Laterza: 3-17.

—. 1993. "La storia 'critica' della filosofia nel Settecento". In Eugenio Garin (ed.), Dal Rinascimento all'Illuminismo. Studi e ricerche. Firenze: Le Lettere.

Geldsetzer, Lutz. 1966. "Der Methodenstreit in der Philosophiegeschichtsschreibung: 1791-1820". Kant-Studien 56: 519-527.

Grafton, Anthony. 2006. "The History of Ideas: Precept and Practice, 19502000 and Beyond".Journal of the History of Ideas 67-1: 1-32.

Graness, Anke. 2015. "Questions of Canon Formation in Philosophy. The History of Philosophy in Africa". Phronimon (Pretoria) 16-2: 78-96.

Hutton, Sarah. 2014. "Intellectual History and the History of Philosophy". History of European Ideas 40-7: 925-937.

Jaran, François. 2011. “Cómo la tradición continental y la tradición analítica se enfrentan con la tradición filosófica”. Revista de Filosofía 36-1 : 171192.

König-Pralong, Catherine. 2016. Médiévisme philosophique et raison moderne de Pierre Bayle à Ernest Renan. Paris: Vrin.

King, Preston (ed.). 1983. The History of Ideas. An Introduction to Method. London: Croom Helm.

Mandelbaum, Maurice. 1965. "The History of Ideas, Intellectual History and the History of Philosophy". in Georg H. Nadel (ed.), The Historiography of the History of Philosophy, History and Theory. Studies in the Philosophy of History, Beiheft 5. Gravenhage: Mouton: 33-66.

Malebranche, Nicolas de. 1946. De la recherche de la vérité. Vol I. Paris: Vrin.

Malik, Kenan. 2017 (Sunday 19 February). "Are Soas [= School of Oriental and African Studies] students right to 'decolonise' their minds from western philosophy?". The Guardian.

Malusa, Luciano. 1993. “The First General Histories of Philosophy in England and the Low Countries". In Giovanni Santinello, Constance Blackwell (eds.), Models of the History of Philosophy, vol. I: From the Origins in the Renaissance to the Historia Philosophica. Dordrecht: Kluwer, 1993 : 236-259.

Merker, Nicolao. 1974. "Per una storiografia filosofica marxista", in Nicolao Merker, Marxismo e storia delle idee. Roma: Editori Riuniti: 13-47.

Micheli, Giuseppe; Santinello, Giovanni; Bianco, Bruno; Longo, Mario. 2015. "Kantianism and the Historiography of Philosophy". In Gregorio Piaia and Giovanni Santinello (eds.), Models of the History of Philosophy, vol. III: The Second Enlightenment and the Kantian Age. Dordrecht: Springer: 769-963.

Moretto, Giovanni. 1979. Etica e storia in Schleiermacher. Napoli: Bibliopolis. Montaigne, Michel de. 1982. Essais, I, 37 (trad. italiana: Saggi, I-II, a cura di Fausta Garavini. Milano: Adelphi). 
Longo, Mario. 1986. Historia philosophiae philosophica. Teorie e metodi della storia della filosofia tra Seicento e Settecento. Milano: IPL: 43-52.

—. 1995. "Ermeneutica e storia della filosofia". in Giovanni Santinello, Gregorio Piaia (eds.), Storia delle storie generali della filosofia, vol. 4/1: 183-348.

-. (ed.). 2003. La storia "generale" della filosofia fra costruzione e decostruzione (Rivista di storia della filosofia 55-2).

—. 2011. "A 'Critical' History of Philosophy and the Early Enlightenment: Johann Jacob Brucker". In Gregorio Piaia, Giovanni Santinello (eds.), Models of the History of Philosophy, vol. II: From the Cartesian Age to Brucker. Dordrecht: Springer: 547-549.

Lovejoy, Arthur. 1940. "Reflections on the History of Ideas". Journal of the History of Ideas 1-1: 3-23.

Luporini, Cesare. 1974. Dialettica e materialismo. Roma: Editori Riuniti.

Richter, Melvin. 1987. "Begriffsgeschichte and the History of Ideas". Journal of the History of Ideas 48-2: 247-263.

Paganini, Gianni. 2014. "Storia della filosofia e storia delle idee filosofiche. Riflessioni di metodo". Philosophia. Rivista della Società Italiana di Storia della Filosofia 10-11.

Park, Peter K. J. 2013. Africa, Asia and the History of Philosophy. Racism in the Formation of Philosophical Canon, 1780-1830. New York: State Univ. of New York Press.

Piaia, Gregorio. 1975. "Storia della filosofia e decolonizzazione del passato". In Storiografia ed ermeneutica. Atti del XIX Convegno di assistenti universitari di filosofia, Padova 1974. Padova: Gregoriana: 47-59.

-.2001. "Brucker versus Rorty? On the 'Models' of the Historiography of Philosophy". British Journal for the History of Philosophy 9-1: 69-81.

-.2004. "Storicismo ed eclettismo: l'età di Victor Cousin". In Giovanni Santinello, Gregorio Piaia, Storia delle storie generali della filosofia, vol. IV/2: L'età hegeliana. La storiografia filosofica nell'area neolatina, danubiana e russa. Roma-Padova: Antenore, 2004 89-200.

Piaia, Gregorio; Santinello, Giovanni (eds.). 2011. Models of the History of Philosophy, vol. II: From the Cartesian Age to Brucker. Dordrecht: Springer.

Piovani, Pietro. 1985. Filosofia e storia delle idee. Bari: Laterza.

Pozzo, Riccardo. 2014. "Storia storica e storia filosofica della filosofia nel XX e nel XXI secolo". Philosophia 10-11: 103-119.

Quine, Willard Van Orman. 1985. The Time of My Life. Cambridge [Ma.]: Mit Press.

Reck, Erich H. (ed.). 2013. The Historical Turn in Analytical Philosophy. Basingstoke: Palgrave Macmillan, 2013).

Rosmini-Serbati, Antonio. 1883. "Sull'unità dell'educazione". In Antonio Rosmini-Serbati, Scritti vari di metodo e di pedagogia. Torino: Unione tipografico-editrice: 53-54. 
Rorty, Richard. 1984. "The Historiography of Philosophy: Four Genres". In Richard Rorty, Jerome B. Schneewind, Quentin Skinner (eds.), Philosophy in History. Essays on Historiography of Philosophy. Cambridge: Cambridge Univ. Press: 49-75.

Rotterdam, Erasmo da. 1997. Elogio della follia (a cura di Carlo Carena). Torino: Einaudi.

Santinello, Giovanni. 1995. "Storia della filosofia e dialettica: Hegel". In Giovanni Santinello e Gregorio Piaia (eds.), Storia delle storie generali della filosofia, vol. IV/1: l'Età hegeliana. La storiografia filosofica nell'area tedesca. Padova: Antenore, 1995: 413-509.

Schleiermacher, Friedrich D. E. 1996. Ermeneutica. Milano: Rusconi.

Scholtz, Gunter. 1996. "Che cos'è e perché ci occupiamo di storia dei concetti?”. Archivio di storia della cultura 9: 133-151.

Sorell, Tom, Rogers, Graham Allan John (eds.). 2005. Analytic Philosophy and the History of Philosophy. Oxford: Clarendon Press.

Stefanini, Luigi. 1962. "Storia, storicità, storicismo, storiografia e persona". In Luigi Stefanini, Personalismo filosofico. Brescia: La Scuola: 47-77.

Tessitore, Fulvio. 1990. Storiografia e storia della cultura. Bologna: Il Mulino.

-.2014. "Storia della filosofia, storia delle idee, storia della cultura filosofica". Philosophia 10-1 (2014): 3-29.

Vegas González, Serafín. 1993. "La revisión neohistoricista del significado de la historia de la filosofía". Anales del Seminario de Historia de la Filosofía 10: 11-42.

Viano, Carlo Augusto. 1966. "Storia della filosofia e sociologia". Rivista di filosofia 57: 255-261.

Vienne, Jean-Michel (ed.). 1997. Philosophie analytique et histoire de la philosophie. Paris: Vrin. 